\title{
Decomposing Cubic Graphs into Connected Subgraphs of Size Three
}

\author{
Laurent Bulteau ${ }^{1}$, Guillaume Fertin ${ }^{2}$, Anthony Labarre ${ }^{1}$, Romeo Rizzi ${ }^{3}$, and \\ Irena Rusu ${ }^{2}$ \\ 1 Université Paris-Est, LIGM (UMR 8049), CNRS, ENPC, ESIEE Paris, UPEM, \\ F-77454, Marne-la-Vallée, France \\ 2 Laboratoire d'Informatique de Nantes-Atlantique, UMR CNRS 6241, Université de \\ Nantes, 2 rue de la Houssinière, 44322 Nantes Cedex 3, France \\ 3 Department of Computer Science, University of Verona, Italy
}

\begin{abstract}
Let $S=\left\{K_{1,3}, K_{3}, P_{4}\right\}$ be the set of connected graphs of size 3. We study the problem of partitioning the edge set of a graph $G$ into graphs taken from any non-empty $S^{\prime} \subseteq S$. The problem is known to be NP-complete for any possible choice of $S^{\prime}$ in general graphs. In this paper, we assume that the input graph is cubic, and study the computational complexity of the problem of partitioning its edge set for any choice of $S^{\prime}$. We identify all polynomial and NP-complete problems in that setting, and give graph-theoretic characterisations of $S^{\prime}$-decomposable cubic graphs in some cases.
\end{abstract}

\section{Introduction}

General context. Given a connected graph $G$ and a set $S$ of graphs, the $S$ DECOMPOSITION problem asks whether $G$ can be represented as an edge-disjoint union of subgraphs, each of which is isomorphic to a graph in $S$. The problem has a long history that can be traced back to Kirkman [7] and has been intensively studied ever since, both from pure mathematical and algorithmic point of views. One of the most notable results in the area is the proof by Dor and Tarsi 3] of the long-standing "Holyer conjecture" [6], which stated that the $S$-DECOMPOSITION problem is NP-complete when $S$ contains a single graph with at least three edges.

Many variants of the $S$-DECOMPOSITION problem have been studied while attempting to prove Holyer's conjecture or to obtain polynomial-time algorithms in restricted cases 12], and applications arise in such diverse fields as traffic grooming [10] and graph drawing [5]. In particular, Dyer and Frieze [4] studied a variant where $S$ is the set of connected graphs with $k$ edges for some natural $k$, and proved the NP-completeness of the $S$-DECOMPOSITION problem for any $k \geq 3$, even under the assumption that the input graph is planar and bipartite (see Theorem 3.1 in [4]). They further claimed that the problem remains NPcomplete under the additional constraint that all vertices of the input graph have degree either 2 or 3 . Interestingly, if one looks at the special case where $k=3$ and $G$ is a bipartite cubic graph (i.e., each vertex has degree 3), then $G$ can clearly be decomposed in polynomial time, using $K_{1,3}$ 's only, by selecting 
either part of the bipartition and making each vertex in that set the center of a $K_{1,3}$. This shows that focusing on the case $k=3$ and on cubic graphs can lead to tractable results - as opposed to general graphs, for which when $k=3$, and for any non empty $S^{\prime} \subseteq S$, the $S^{\prime}$-DECOMPOSITION problems all turn out to be NP-complete [4, 6].

In this paper, we study the $S$-DECOMPOSITION problem on cubic graphs in the case $k=3$ - i.e., $S=\left\{K_{1,3}, K_{3}, P_{4}\right\}$. For any non-empty $S^{\prime} \subseteq S$, we settle the computational complexity of the $S^{\prime}$-DECOMPOSITION problem by showing that the problem is NP-complete when $S^{\prime}=\left\{K_{1,3}, P_{4}\right\}$ and $S^{\prime}=S$, while all the other cases are in P. Table 1 summarises the state of knowledge regarding the complexity of decomposing cubic and arbitrary graphs using connected subgraphs of size three, and puts our results into perspective.

\begin{tabular}{|c|c|c||c|c|}
\hline \multicolumn{3}{|c||}{ Allowed subgraphs } & \multicolumn{2}{c|}{ Complexity according to graph class } \\
\hline$K_{1,3}$ & $K_{3}$ & $P_{4}$ & cubic & arbitrary \\
\hline \hline$\checkmark$ & & & in P (Proposition 3) & NP-complete [4, Theorem 3.5] \\
& $\checkmark$ & & N (1) (impossible) & NP-complete [6] \\
& & $\checkmark$ & in P [8] & NP-complete [4, Theorem 3.4] \\
\hline$\checkmark$ & $\checkmark$ & & in P (Proposition 6) & NP-complete [4, Theorem 3.5] \\
$\checkmark$ & & $\checkmark$ & NP-complete (Theorem 2) & NP-complete [4, Theorem 3.1] \\
& $\checkmark$ & $\checkmark$ & in P (Proposition 2) & NP-complete [4, Theorem 3.4] \\
\hline$\checkmark$ & $\checkmark$ & $\checkmark$ & NP-complete (Theorem $\underline{\text { 3) }}$ & NP-complete [4, Theorem 3.1] \\
\hline
\end{tabular}

Table 1. Known complexity results on decomposing graphs using subsets of $\left\{K_{1,3}, K_{3}, P_{4}\right\}$.

Terminology. We follow Brandstädt et al. 2] for notation and terminology. All graphs we consider are simple, connected and nontrivial (i.e. $|V(G)| \geq 2$ and $|E(G)| \geq 1$ ). Given a set $S$ of graphs, a graph $G$ admits an $S$-decomposition, or is $S$-decomposable, if $E(G)$ can be partitioned into subgraphs, each of which is isomorphic to a graph in $S$. Throughout the paper, $S$ denotes the set of connected graphs of size 3, i.e. $S=\left\{K_{3}, K_{1,3}, P_{4}\right\}$. We study the following problem:

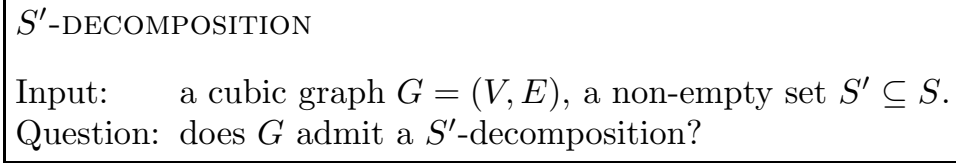

We let $G[U]$ denote the subgraph of $G$ induced by $U \subseteq V(G)$. Given a graph $G=(V, E)$, removing a subgraph $H=\left(V^{\prime} \subseteq V, E^{\prime} \subseteq E\right)$ of $G$ consists in removing edges $E^{\prime}$ from $G$ as well as the possibly resulting isolated vertices. Finally, let $G$ and $G^{\prime}$ be two graphs. Then:

- subdividing an edge $\{u, v\} \in E(G)$ consists in inserting a new vertex $w$ into that edge, so that $V(G)$ becomes $V(G) \cup\{w\}$ and $E(G)$ is replaced with $E(G) \backslash\{u, v\} \cup\{u, w\} \cup\{w, v\} ;$

- attaching $G^{\prime}$ to a vertex $u \in V(G)$ means building a new graph $H$ by identifying $u$ and some $v \in V\left(G^{\prime}\right)$; 
- attaching $G^{\prime}$ to an edge $e \in E(G)$ consists in subdividing $e$ using a new vertex $w$, then attaching $G^{\prime}$ to $w$.

Figure 1 illustrates the process of attaching an edge to an edge of the cube graph, and shows other small graphs that we will occasionally use in this paper.

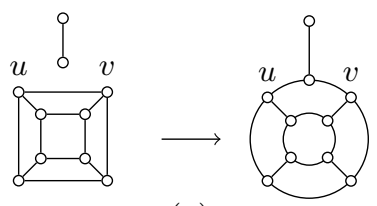

(a)

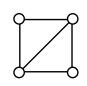

(b)

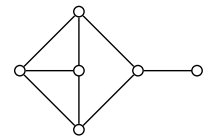

(c)

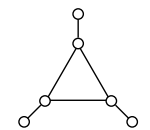

(d)

Fig. 1. (a) Attaching a new edge to $\{u, v\} ;(b)$ the diamond graph; (c) the co-fish graph; $(d)$ the net graph.

\section{Decompositions Without a $K_{1,3}$}

In this section, we study decompositions of cubic graphs that use only $P_{4}$ 's or $K_{3}$ 's. Note that no cubic graph is $\left\{K_{3}\right\}$-decomposable, since all its vertices have odd degree. According to Bouchet and Fouquet [1], Kotzig [8] proved that a cubic graph admits a $\left\{P_{4}\right\}$-decomposition iff it has a perfect matching. However, the proof of the forward direction as presented in [1] is incomplete, as it requires the use of Proposition 1. (b) below, which is missing from their paper. Therefore, we provide the following proposition for completeness, together with another result which will also be useful for the case where $S^{\prime}=\left\{K_{3}, P_{4}\right\}$.

Proposition 1. Let $G$ be a cubic graph that admits a $\left\{K_{3}, P_{4}\right\}$-decomposition $D$. Then, in $D$, (a) no $K_{3}$ is used, and (b) no three $P_{4}$ 's are incident to the same vertex.

Proof. Partition $V(G)$ into three sets $V_{1}, V_{2}$ and $V_{3}$, where $V_{1}$ (resp. $V_{2}, V_{3}$ ) is the set of vertices that are incident to exactly one $P_{4}$ (resp. two, three $P_{4}$ 's) in $D$. Note that $V_{1}$ is exactly the set of vertices involved in $K_{3}$ 's in $D$. Let $n_{i}=\left|V_{i}\right|$, $1 \leq i \leq 3$. Our goal is to show that $n_{1}=n_{3}=0$, i.e. $V_{1}=V_{3}=\emptyset$. For this, note that (1) each vertex in $V_{3}$ is the extremity of three different $P_{4}$ 's, (2) each vertex in $V_{2}$ is simultaneously the extremity of one $P_{4}$ and an inner vertex of another $P_{4}$, while (3) each vertex in $V_{1}$ is the extremity of one $P_{4}$. Since each $P_{4}$ has two extremities and two inner vertices, if $p$ is the number of $P_{4}$ 's in $D$, we have:

- $p=\frac{3 n_{3}+n_{2}+n_{1}}{2}$ (by (1), (2) and (3) above, counting extremities);

- $p=\frac{n_{2}}{2}$ (by (2) above, counting inner vertices).

Putting together the above two equalities yields $n_{1}=n_{3}=0$, which completes the proof. 
Since $K_{3}$ 's cannot be used in cubic graphs for $\left\{K_{3}, P_{4}\right\}$-decompositions by Proposition 1 above, we directly obtain the following result, which implies that $\left\{K_{3}, P_{4}\right\}$-decomposition is in $\mathrm{P}$.

Proposition 2. A cubic graph admits a $\left\{K_{3}, P_{4}\right\}$-decomposition iff it has a perfect matching.

\section{Decompositions Without a $\boldsymbol{P}_{4}$}

In this section, we study decompositions of cubic graphs that use only $K_{1,3}$ 's or $K_{3}$ 's.

Proposition 3. A cubic graph $G$ admits a $\left\{K_{1,3}\right\}$-decomposition iff it is bipartite.

Proof. For the reverse direction, select either set of the bipartition, and make each vertex in that set the center of a $K_{1,3}$. For the forward direction, let $D$ be a $\left\{K_{1,3}\right\}$-decomposition of $G$, and let $C$ and $L$ be the sets of vertices containing, respectively, all the centers and all the leaves of $K_{1,3}$ 's in $D$. We show that this is a bipartition of $V(G)$. First, $C \cup L=V$ since $D$ covers all edges and therefore all vertices. Second, $C \cap L=\emptyset$ since a vertex in $C \cap L$ would have degree at least 4. Finally, each edge in $D$ connects the center of a $K_{1,3}$ and a leaf of another $K_{1,3}$ in $D$, which belong respectively to $C$ and $L$. Therefore, $G$ is bipartite.

We now prove that $\left\{K_{1,3}, K_{3}\right\}$-decompositions can be computed in polynomial time. Recall that a graph is $H$-free if it does not contain an induced subgraph isomorphic to a given graph $H$. Since bipartite graphs admit a $\left\{K_{1,3}\right\}$ decomposition (by Proposition 3 ), we can restrict our attention to non-bipartite graphs that contain $K_{3}$ 's (indeed, if they were $K_{3}$-free, then only $K_{1,3}$ 's would be allowed and Proposition 3 would imply that they admit no decomposition). Our strategy consists in iteratively removing subgraphs from $G$ and adding them to an initially empty $\left\{K_{1,3}, K_{3}\right\}$-decomposition until $G$ is empty, in which case we have an actual decomposition, or no further removal operations are possible, in which case no decomposition exists. Our analysis relies on the following notion: a $K_{3}$ induced by vertices $\{u, v, w\}$ in a graph $G$ is isolated if $V(G)$ contains no vertex $x$ such that $\{u, v, x\},\{u, x, w\}$ or $\{x, v, w\}$ induces a $K_{3}$.

Lemma 1. If a cubic graph $G$ admits a $\left\{K_{1,3}, K_{3}\right\}$-decomposition $D$, then every isolated $K_{3}$ in $G$ belongs to $D$.

Proof (contradiction). If an isolated $K_{3}$ were not part of the decomposition, then exactly one vertex of that $K_{3}$ would be the center of a $K_{1,3}$, leaving the remaining edge uncovered and uncoverable.

$\overline{C_{6}}$ is a minimal example of a cubic non-bipartite graph with $K_{3}$ 's that admits no $\left\{K_{1,3}, K_{3}\right\}$-decomposition: both $K_{3}$ 's in that graph must belong to the decomposition (by Lemma 1), but their removal yields a perfect matching. 
Observation 1. Let $G$ be a connected cubic graph. Then no sequence of at least one edge or vertex removal from $G$ yields a cubic graph.

Proof (contradiction). If after applying at least one removal from $G$ we obtain a cubic graph $G^{\prime}$, then the graph that precedes $G^{\prime}$ in this removal sequence must have had a vertex of degree at least four, since $G$ is connected.

Proposition 4. For any non-bipartite cubic graph $G$ whose $K_{3}$ 's are all isolated, one can decide in polynomial time whether $G$ is $\left\{K_{1,3}, K_{3}\right\}$-decomposable.

Proof. We build a $\left\{K_{1,3}, K_{3}\right\}$-decomposition by iteratively removing $K_{1,3}$ 's and $K_{3}$ 's from $G$, which we add as we go to an initially empty set $D$. By Lemma 1 , all isolated $K_{3}$ 's must belong to $D$, so we start by adding them all to $D$ and removing them from $G$; therefore, $G$ admits a $\left\{K_{1,3}, K_{3}\right\}$-decomposition iff the resulting subcubic graph $G^{\prime}$ admits a $\left\{K_{1,3}\right\}$-decomposition. Observe that $G^{\prime}$ contains vertices of degree 1 and 2 ; we note that:

1. each vertex of degree 1 must be the leaf of some $K_{1,3}$ in $D$;

2. each vertex of degree 2 must be the meeting point of two $K_{1,3}$ 's in $D$.

The only ambiguity arises for vertices of degree 3 , which may either be the center of a $K_{1,3}$ in $D$ or the meeting point of three $K_{1,3}$ 's in $D$; however, there will always exist at least one other vertex of degree 1 or 2 until the graph is empty (by Observation 1.). Therefore, we can safely remove $K_{1,3}$ 's from our graph and add them to $D$ by following the above rules in the stated order; if we succeed in deleting the whole graph in this way, then $D$ is a $\left\{K_{1,3}, K_{3}\right\}$-decomposition of $G$, otherwise no such decomposition exists.

We conclude with the case where the graph may contain non-isolated $K_{3}$ 's.

Proposition 5. If a cubic graph $G$ contains a diamond, then one can decide in polynomial time whether $G$ is $\left\{K_{1,3}, K_{3}\right\}$-decomposable.

Proof. The only cubic graph on 4 vertices is $K_{4}$, which is diamond-free and $\left\{K_{1,3}, K_{3}\right\}$-decomposable, so we assume $|V(G)| \geq 6$. Let $\mathcal{D}$ be a diamond in $G$ induced by vertices $\{u, v, w, x\}$ and such that $\{u, x\} \notin E(G)$, as shown in Figure $2(a)$. $\mathcal{D}$ is connected to two other vertices $u^{\prime}$ and $x^{\prime}$ of $G$, which are respectively adjacent to $u$ and $x$, and there are only two ways to use the edges of $\mathcal{D}$ in a $\left\{K_{1,3}, K_{3}\right\}$-decomposition, as shown in Figure $2(b)$ and $(c)$. If $u^{\prime}=x^{\prime}$, regardless of the decomposition we choose for $\mathcal{D}, u^{\prime}$ and its neighbourhood induce a $P_{3}$ in the graph obtained from $G$ by removing the parts added to $D$. But then that $P_{3}$ cannot be covered, so no $\left\{K_{1,3}, K_{3}\right\}$-decomposition exists for $G$. Therefore, we assume that $u^{\prime} \neq x^{\prime}$.

As Figure 2(b) and (c) show, either $\{u, v, w\}$ or $\{v, w, x\}$ must form a $K_{3}$ in $D$, thereby forcing either $\left\{v, w, x, x^{\prime}\right\}$ or $\left\{u^{\prime}, u, v, w\right\}$ to form a $K_{1,3}$ in $D$. In both cases, removing the $K_{3}$ and the $K_{1,3}$ yields a graph $G^{\prime}$ which contains vertices of degree 1,2 or 3. As in the proof of Proposition 4, Observation 1 allows us to make the following helpful observations: 
1. every leaf in $G^{\prime}$ must be the leaf of some $K_{1,3}$ in $D$;

2. every vertex $y$ of degree two in $G^{\prime}$ must either belong to a $K_{3}$ or be a leaf of two distinct $K_{1,3}$ 's in $D$, which can be decided as follows:

(a) if $y$ belongs to a $K_{3}$ in $G^{\prime}$, then it must also belong to a $K_{3}$ in $D$; otherwise, it would be the leaf of a $K_{1,3}$ and the graph obtained by removing that $K_{1,3}$ would contain a $P_{3}$, which we cannot cover;

(b) otherwise, $y$ must be a leaf of two $K_{1,3}$ 's in $D$.

We therefore iteratively remove subgraphs from our graph and add them to $D$ according to the above rules, which we follow in the stated order; if we succeed in deleting the whole graph in this way using either decomposition in Figure 2 $(b)$ or $(c)$ as a starting point, then $D$ is a $\left\{K_{1,3}, K_{3}\right\}$-decomposition of $G$, otherwise no such decomposition exists.

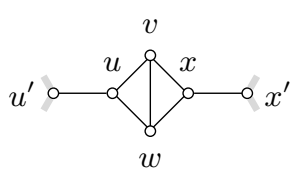

(a)

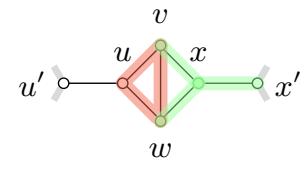

(b)

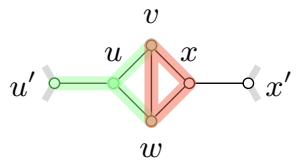

(c)

Fig. 2. (a) A diamond in a cubic graph, and $(b),(c)$ the only two ways to decompose it in a $\left\{K_{1,3}, K_{3}\right\}$-decomposition.

All the arguments developed in this section lead to the following result.

Proposition 6. The $\left\{K_{1,3}, K_{3}\right\}$-DECOMPOSITION problem on cubic graphs is in $\mathrm{P}$.

\section{Decompositions That Use Both $K_{1,3}$ 's and $P_{4}$ 's}

In this section, we show that problems $\left\{K_{1,3}, P_{4}\right\}$-DECOMPOSITION and $\left\{K_{1,3}\right.$, $\left.K_{3}, P_{4}\right\}$-Decomposition are NP-complete. Our hardness proof relies on two intermediate problems that we define below and is structured as follows:

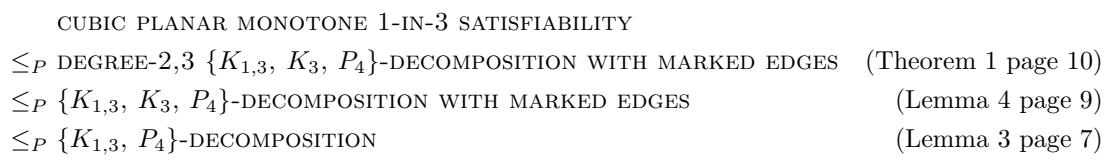

We start by introducing the following intermediate problem:

$\left\{K_{1,3}, K_{3}, P_{4}\right\}$-DECOMPOSITION WITH MARKED EDGES

Input: $\quad$ a cubic graph $G=(V, E)$ and a subset $M \subseteq E$ of edges.

Question: does $G$ admit a $\left\{K_{1,3}, K_{3}, P_{4}\right\}$-decomposition $D$ such that no edge in $M$ is the middle edge of a $P_{4}$ in $D$ and such that every $K_{3}$ in $D$ has either one or two edges in $M$ ? 
The drawings that illustrate our proofs in this section show marked edges as dotted edges. The proof of Lemma 3 uses the following result.

Lemma 2. Let e be a bridge in a cubic graph $G$ which admits a $\left\{K_{1,3}, K_{3}, P_{4}\right\}$ decomposition $D$. Then e must be the middle edge of a $P_{4}$ in $D$.

Proof (contradiction). First note that $e$ cannot belong to a $K_{3}$ in $D$. Now suppose $e$ is part of a $K_{1,3}$ in $D$. The situation is as shown below (without loss of generality):

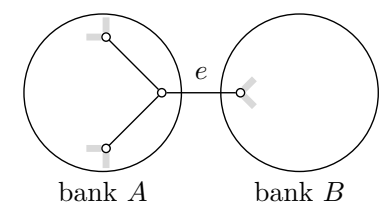

If we remove from $G$ the $K_{1,3}$ in $D$ that contains $e$, then summing the terms of the degree sequence of $G[V(B)]$ yields $2+3(|V(B)|-1)=2|E(B)|$, which means that $2|E(B)| \equiv 2(\bmod 3)$, so $|E(B)| \not \equiv 0(\bmod 3)$ and therefore $B$ admits no decomposition into components of size three. The very same argument shows that if $e$ belongs to a $P_{4}$ in $D$, then it must be its middle edge, which completes the proof.

Lemma 3. Let $(G, M)$ be an instance of $\left\{K_{1,3}, K_{3}, P_{4}\right\}$-DECOMPOSITION With MARKED EDGES, and $G^{\prime}$ be the graph obtained by attaching a co-fish to every edge in $M$. Then $G$ can be decomposed iff $G^{\prime}$ admits a $\left\{K_{1,3}, P_{4}\right\}$-decomposition.

Proof. We prove each direction separately.

$\Rightarrow$ : we show how to transform a decomposition $D$ of $(G, M)$ into a decomposition $D^{\prime}$ of $G^{\prime}$. The subgraphs in $D$ that have no edge in $M$ are not modified. For the other subgraphs, we distinguish between four cases:

(a) if an edge of $M$ belongs to a $K_{1,3}$ in $D$, then attaching a co-fish does not prevent us from adapting the decomposition of $G$ in $G^{\prime}$ :

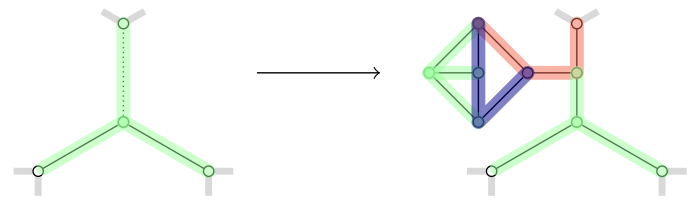

(b) if an edge of $M$ belongs to a $P_{4}$ in $D$, then it is an extremity of that $P_{4}$ and attaching a co-fish does not prevent us from adapting that part of the decomposition:

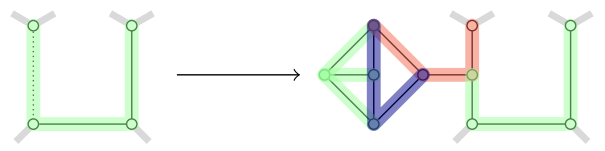

(c) if a $K_{3}$ in $D$ has one edge in $M$, we can adapt the partition as follows: 


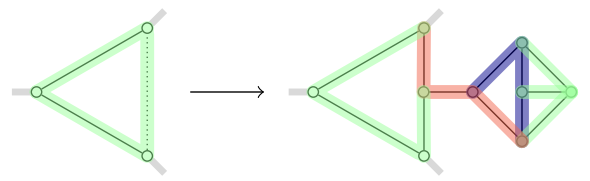

(d) if a $K_{3}$ in $D$ has two edges in $M$, we can adapt the partition as follows:

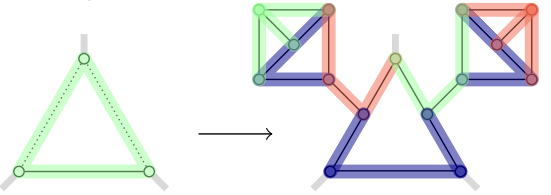

$\Leftarrow$ : we now show how to transform any $\left\{K_{1,3}, P_{4}\right\}$-decomposition $D^{\prime}$ of $G^{\prime}$ into a decomposition of $(G, M)$. Again, the only parts of $D^{\prime}$ that will need adapting are those connected to the co-fishes that we inserted when transforming $G$ into $G^{\prime}$. Since the leaf $u$ of the co-fish we inserted has a neighbour $x$ such that $\{u, x\}$ is a bridge in $G^{\prime},\{u, x\}$ is the middle edge of a $P_{4}$ in $D^{\prime}$ (Lemma 2) and we may therefore assume without loss of generality that our starting point in $G^{\prime}$ is as follows:

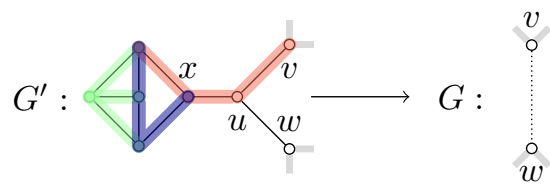

with $\{v, w\} \notin E\left(G^{\prime}\right)$ since $G$ is simple; therefore $\{u, w\}$ cannot belong to a $K_{3}$ in $G^{\prime}$, and we have two cases to consider:

(a) if $\{u, w\}$ belongs to a $K_{1,3}$ in $D^{\prime}$, that $K_{1,3}$ can be mapped onto a $K_{1,3}$ in $D$ by replacing $\{u, w\}$ with $\{v, w\}$;

(b) otherwise, $\{u, w\}$ is an extremal edge of a $P_{4}$ in $D^{\prime}$; since $\{u, w\} \notin E(G)$, either that edge will remain in a $P_{4}$ when removing the co-fish and replacing $\{u, w\}$ with $\{v, w\}$, or it will end up in a $K_{3}$ with either one or two marked edges. Either way, the part can be added as such to $D$.

We now show that we can restrict our attention to the following variant of $\left\{K_{1,3}, K_{3}, P_{4}\right\}$-DECOMPOSITION With MARKED EDGES. We say a graph is degree-2,3 if its vertices have degree only 2 or 3 .

DEGREE- $2,3\left\{K_{1,3}, K_{3}, P_{4}\right\}$-DECOMPOSITION WITH MARKED EDGES

Input: $\quad$ a degree-2,3 graph $G=(V, E)$ and a subset $M \subseteq E$ of edges.

Question: does $G$ admit a $\left\{K_{1,3}, K_{3}, P_{4}\right\}$-decomposition $D$ such that no edge in $M$ is the middle edge of a $P_{4}$ in $D$ and such that every $K_{3}$ in $D$ has either one or two edges in $M$ ?

The following observation will help.

Observation 2. Let $G$ be a degree-2,3 graph with $\left|V_{2}\right|$ degree-2 vertices. If $G$ is $\left\{K_{1,3}, K_{3}, P_{4}\right\}$-decomposable, then $\left|V_{2}\right| \equiv 0(\bmod 3)$. 


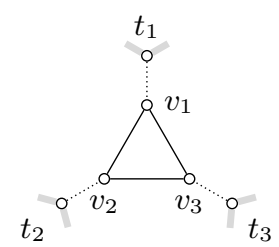

(a)

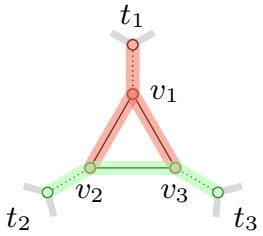

(b)

Fig. 3. Adding a net $(a)$ to a graph with degree- 2 vertices $t_{1}, t_{2}, t_{3}$ (dotted edges belong to $M^{\prime}$ ), and (b) its only possible decomposition (up to symmetry).

Proof. If $G=(V, E)$ admits a $\left\{K_{1,3}, K_{3}, P_{4}\right\}$-decomposition, then $|E| \equiv 0$ $(\bmod 3)$. Let $V_{2}$ and $V_{3}$ be the subsets of vertices of degree 2 and 3 in $G$. Then $2\left|V_{2}\right|+3\left|V_{3}\right|=2|E|$, so $2\left|V_{2}\right| \equiv 0(\bmod 3)$.

We prove that allowing degree-2 vertices does not make the problem substantially more difficult, by adding the following gadgets until all vertices have degree 3 . Let $(G, M)$ be an instance of DEGREE- $2,3\left\{K_{1,3}, K_{3}, P_{4}\right\}$-DECOMPOSITION With MARKED EDGES, where $G$ has at least three degree- 2 vertices $t_{1}, t_{2}, t_{3}$; by adding a net over $\left\{t_{1}, t_{2}, t_{3}\right\}$, we mean attaching a net by its leaves to $v_{1}, v_{2}$ and $v_{3}$ and adding the edges incident to the net's leaves to $M$ (see Figure $3(a)$ ).

Proposition 7. Let $(G, M)$ be an instance of DEGREE- $2,3\left\{K_{1,3}, K_{3}, P_{4}\right\}$-DECOMPOSITION WITH MARKED EDGES, where $G$ has at least three degree-2 vertices $t_{1}, t_{2}, t_{3}$, and let $\left(G^{\prime}, M^{\prime}\right)$ be the instance obtained by adding a net to $(G, M)$. Then $\left(G^{\prime}, M^{\prime}\right)$ has three degree-2 vertices less than $(G, M)$, and $(G, M)$ can be decomposed iff $\left(G^{\prime}, M^{\prime}\right)$ can be decomposed.

Proof. By construction, $G^{\prime}$ has fewer degree-2 vertices, since $t_{1}, t_{2}, t_{3}$ now have degree 3 instead of 2 , other vertices of $G$ are unchanged, and new vertices $\left\{v_{1}, v_{2}, v_{3}\right\}$ have degree 3 . We now prove the equivalence.

$\Rightarrow$ : given a decomposition $D$ for $(G, M)$, we only need to add the $K_{1,3}$ induced by $\left\{v_{1}, t_{1}, v_{2}, v_{3}\right\}$ and the $P_{4}$ induced by $\left\{t_{2}, v_{2}, v_{3}, t_{3}\right\}$ to cover the edges of the added net in order to obtain a decomposition $D^{\prime}$ for $\left(G^{\prime}, M^{\prime}\right)$ (see Figure $3(b))$.

$\Leftarrow$ : we show that the only valid decompositions must include the choice we made in the proof of the forward direction. Indeed, the marked edges cannot be middle edges in a $P_{4}$, and the $K_{3}$ induced by $v_{1}, v_{2}$ and $v_{3}$ cannot appear as a $K_{3}$ in a decomposition. Moreover, no marked edge can be the extremity of a $P_{4}$ with two edges lying in the $K_{3}$, since this would force another marked edge to be the middle edge of a $P_{4}$. Therefore the only possible decomposition of the net is the one defined above (up to symmetry), and we can safely remove the $P_{4}$ and the $K_{1,3}$ from $D^{\prime}$ while preserving the rest of the decomposition.

Lemma 4. DEGREe- $2,3\left\{K_{1,3}, K_{3}, P_{4}\right\}$-DECOMPosition With MARKED EdGes $\leq_{P}\left\{K_{1,3}, K_{3}, P_{4}\right\}$-DECOMPOSITION With MARKED EDGES. 
Proof. Given an instance $(G, M)$ of DEGREe- $2,3\left\{K_{1,3}, K_{3}, P_{4}\right\}$-DECOMPosiTION WITH MARKED EDGES, create an instance $\left(G^{\prime}, M^{\prime}\right)$ by successively adding a net to any triple of degree-2 vertices, until no such triple remains. By Proposition 7, $(G, M)$ is decomposable iff $\left(G^{\prime}, M^{\prime}\right)$ is decomposable. Moreover, either $G^{\prime}$ is cubic (hence $\left(G^{\prime}, M^{\prime}\right)$ is an instance of $\left\{K_{1,3}, K_{3}, P_{4}\right\}$-DECOMPOSITION WITH MARKED EDGES), or $G$ is trivially a no-instance by Observation 2 .

Finally, we show that DEGREe- $2,3\left\{K_{1,3}, K_{3}, P_{4}\right\}$-DECOMPOSition with MARKED EDGES is NP-complete. Our reduction relies on the CUBIC PLANAR MONOTONE 1-IN-3 SATISFIABILITY problem [9]:

CUBIC PLANAR MONOTONE 1-IN-3 SATISFIABILITY

Input: $\quad$ a Boolean formula $\phi=C_{1} \wedge C_{2} \wedge \cdots \wedge C_{n}$ without negations over a set $\Sigma=\left\{x_{1}, x_{2}, \ldots, x_{m}\right\}$, with exactly three distinct variables per clause and where each literal appears in exactly three clauses; moreover, the graph with clauses and variables as vertices and edges joining clauses and the variables they contain is planar.

Question: does there exist an assignment of truth values $f: \Sigma \rightarrow\{$ TRUE, FALSE $\}$ such that exactly one literal is TRUE in every clause of $\phi$ ?

Theorem 1. DEGREe- $2,3\left\{K_{1,3}, K_{3}, P_{4}\right\}$-DECOMPosition With MARKED EDGES is NP-complete.

Proof. We first show how to transform an instance $\phi=C_{1} \wedge C_{2} \wedge \cdots \wedge C_{n}$ of CUBIC PLANAR MONOTONE 1-IN-3 SATISFIABILITY into an instance $(G, M)$ of Degree- $2,3\left\{K_{1,3}, K_{3}, P_{4}\right\}$-DeComposition with marked EDGES. The transformation proceeds by:

1. mapping each variable $x_{i}$ onto a $K_{1,3}$ denoted by $K\left(x_{i}\right)$ and whose edges all belong to $M$;

2. mapping each clause $C=\left\{x_{i}, x_{j}, x_{k}\right\}$ onto a cycle with five vertices in such a way that $K\left(x_{i}\right), K\left(x_{j}\right)$ and $K\left(x_{k}\right)$ each have a leaf that coincides with a vertex of the cycle and exactly two such leaves are adjacent in the cycle.

Figure 4 illustrates the construction, which yields a degree-2,3 graph. We now show that $\phi$ is satisfiable iff $(G, M)$ admits a decomposition.

$\Rightarrow$ : we apply the following rules for transforming a satisfying assignment for $\phi$ into a decomposition $D$ for $(G, M)$ :

- if variable $x_{i}$ is set to FALSE, then the corresponding $K\left(x_{i}\right)$ is added as such to $D$;

- otherwise, the three edges of $K\left(x_{i}\right)$ will be the meeting points of three different $K_{1,3}$ 's in the decomposition, one of which will have two edges in the current clause gadget.

Two cases can be distinguished based on whether or not a leaf of $K\left(x_{i}\right)$ is adjacent to a leaf of $K\left(x_{j}\right)$ or $K\left(x_{k}\right)$, but in both cases the rest of the clause gadget yields a $P_{4}$ that we add as such to the decomposition (see Figure $4(b)$ and $(c))$. 
$\Leftarrow$ : we now show how to convert a decomposition $D$ for $(G, M)$ into a satisfying truth assignment for $\phi$. First, we observe that $D$ must satisfy the following crucial structural property:

For each clause $C=\left(x_{i} \vee x_{j} \vee x_{k}\right)$, exactly two subgraphs out of $K\left(x_{i}\right), K\left(x_{j}\right)$ and $K\left(x_{k}\right)$ appear as $K_{1,3}$ 's in $D$.

Indeed, $G$ is $K_{3}$-free by construction, and:

(a) if all of them appear as $K_{1,3}$ 's in $D$, then the remaining five edges of the clause gadget cannot be decomposed;

(b) if only $K\left(x_{i}\right)$ appear as a $K_{1,3}$ in $D$, then $x_{j}$ - without loss of generality - must be a leaf either of a $K_{1,3}$ in $D$ with a center in the clause gadget or of a $P_{4}$ in $D$ with two edges in the clause gadget (the $P_{4}$ cannot connect $x_{j}$ and $x_{k}$, otherwise the rest of the gadget cannot be decomposed); in both cases, the remaining three edges of the clause gadget must form a $P_{4}$, thereby causing $K\left(x_{k}\right)$ to appear as a $K_{1,3}$ in $D$, a contradiction (a similar argument allows us to handle $K\left(x_{j}\right)$ and $\left.K\left(x_{k}\right)\right)$;

(c) finally, if none of them appear as $K_{1,3}$ 's in $D$, then $x_{i}$ must be the leaf either of a $K_{1,3}$ in $D$ with a center in the clause gadget, or of a $P_{4}$ with two edges in the clause gadget; in both cases, the remaining three edges of the clause gadget must form a $P_{4}$ in $D$, which in turn makes it impossible to decompose the rest of the graph.

Therefore, $D$ yields a satisfying assignment for $\phi$ in the following simple way: if $K\left(x_{i}\right)$ appears as a $K_{1,3}$ in $D$, set it to FALSE, otherwise set it to TRUE.

Theorem 2. $\left\{K_{1,3}, P_{4}\right\}$-DECOMPosition is NP-complete.

Proof. Immediate from Lemmas 3 and 4 and Theorem 1 .

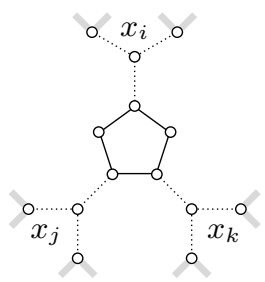

(a)

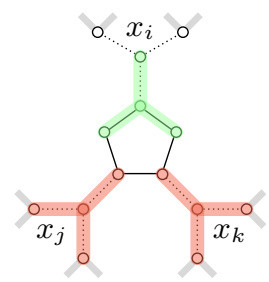

(b)

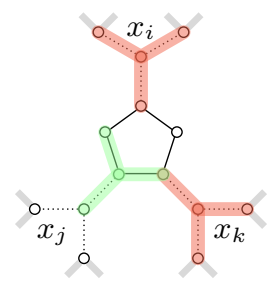

$(c)$

Fig. 4. (a) Connecting clause and variable gadgets in the proof of Theorem 1; dotted edges belong to $M_{\text {. }}(b),(c)$ Converting truth assignments into decompositions in the proof of Theorem 1; the only variable set to TRUE is mapped onto a $K_{1,3}$ in the decomposition; (b) shows the case where the only variable set to TRUE - namely, $x_{i}$ — is such that $K\left(x_{i}\right)$ has no leaf adjacent to a leaf of $K\left(x_{j}\right)$ nor $K\left(x_{k}\right) ;(c)$ shows the other case, where $x_{j}$ is set to TRUE and $K\left(x_{i}\right)$ and $K\left(x_{k}\right)$ have leaves made adjacent by the clause gadget. 
A like-minded reduction 11 allows us to prove the hardness of $\left\{K_{1,3}, K_{3}, P_{4}\right\}$ DECOMPOSITION.

Theorem 3. $\left\{K_{1,3}, K_{3}, P_{4}\right\}$-DECOMPOSITION is NP-complete, even on $K_{3}$-free graphs.

\section{Conclusions and Future Work}

We provided in this paper a complete complexity landscape of $\left\{K_{1,3}, K_{3}, P_{4}\right\}$ DECOMPOSITION for cubic graphs. A natural generalisation, already studied by other authors, is to study decompositions of $k$-regular graphs into connected components with $k$ edges for $k>3$. We would like to determine whether our positive results generalise in any way in that setting. It would also be interesting to identify tractable classes of graphs in the cases where those decomposition problems are hard, and to refine our characterisation of hard instances; for instance, does there exist a planarity-preserving reduction for Theorem 3 ? Finally, we note that some applications relax the size constraint by allowing the use of graphs with at most $k$ edges in the decomposition [10]; we would like to know how that impacts the complexity of the problems we study in this paper.

\section{References}

[1] A. Bouchet And J.-L. Fouquet, Trois types de décompositions d'un graphe en chaînes, in Combinatorial Mathematics: Proceedings of the International Colloquium on Graph Theory and Combinatorics, C. Berge, D. Bresson, P. Camion, J. F. Maurras, and F. Sterboul, eds., vol. 75 of North-Holland Mathematics Studies, North-Holland, 1983, pp. 131-141.

[2] A. Brandstädt, V. B. Le, And J. P. Spinrad, Graph classes: a survey, SIAM Monographs on Discrete Mathematics and Applications, Society for Industrial Mathematics, 1987.

[3] D. Dor And M. TARsI, Graph decomposition is NP-complete: A complete proof of Holyer's conjecture, SIAM J. Comput., 26 (1997), pp. 1166-1187.

[4] M. E. Dyer AND A. M. Frieze, On the complexity of partitioning graphs into connected subgraphs, Discrete Appl. Math., 10 (1985), pp. 139-153.

[5] É. FusY, Transversal structures on triangulations: A combinatorial study and straight-line drawings, Discrete Math., 309 (2009), pp. 1870-1894.

[6] I. HolyER, The NP-completeness of some edge-partition problems, SIAM J. Comput., 10 (1981), pp. 713-717.

[7] T. P. Kirkman, On a problem in combinatorics, Cambridge Dublin Mathematical Journal, 2 (1847), pp. 191-204.

[8] A. Kotzig, Z teorie konečných pravidelných grafov tretieho a štvrtého stupña, Časopis pro pěstování matematiky, (1957), pp. 76-92.

[9] C. Moore And J. M. Robson, Hard tiling problems with simple tiles, Discrete Comput. Geom., 26 (2001), pp. 573-590.

\footnotetext{
${ }^{1}$ See Appendix for details.
} 
[10] X. MuÑoz, Z. LI, AND I. SAU, Edge-partitioning regular graphs for ring traffic grooming with a priori placement of the ADMs, SIAM J. Discrete Math., 25 (2011), pp. 1490-1505.

[11] T. J. Schaefer, The complexity of satisfiability problems, in Proc. 10th STOC, San Diego, California, USA, May 1978, ACM, pp. 216-226.

[12] R. Yuster, Combinatorial and computational aspects of graph packing and graph decomposition, Computer Science Review, 1 (2007), pp. 12-26. 


\section{A Appendix: Omitted Proofs}

Our hardness proof uses ideas similar to those used for $\left\{K_{1,3}, P_{4}\right\}$-decomposition, and is based on a slightly different intermediate problem. The structure is as follows:

$$
\begin{aligned}
& \text { MONOTONE NOT-ALL-EQUAL 3-SATISFIABILITY } \\
& \leq_{P} K_{3} \text {-Free }\left\{K_{1,3}, P_{4}\right\} \text {-DECOMPosition With MARKed EDGES (Theorem } 4 \text { page 14) } \\
& \leq_{P}\left\{K_{1,3}, K_{3}, P_{4}\right\} \text {-DECOMPosition } \quad \text { (Lemma } 5 \text { page 14) }
\end{aligned}
$$

We use the following intermediate problem.

$K_{3}$-FreE $\left\{K_{1,3}, P_{4}\right\}$-DECOMPOSITION With MARKED EDGeS

Input: $\quad$ a cubic, $K_{3}$-free graph $G=(V, E)$ and a subset $M \subseteq E$ of edges.

Question: does $G$ admit a $\left\{K_{1,3}, P_{4}\right\}$-decomposition $D$ such that no edge in $M$ is the middle edge of a $P_{4}$ in $D$ ?

Lemma 5. Let $(G, M)$ be an instance of $K_{3}$-FREE $\left\{K_{1,3}, P_{4}\right\}$-DECOMPOSITION WITH MARKED EDGES, and $G^{\prime}$ be the graph obtained by attaching a co-fish to every edge in $M$. Then $G$ can be decomposed iff $G^{\prime}$ admits a $\left\{K_{1,3}, K_{3}, P_{4}\right\}$-decomposition.

Proof. The proof of the forward direction is exactly the same as that of the forward direction of Lemma 3. For the reverse direction, let $D^{\prime}$ be a $\left\{K_{1,3}\right.$, $\left.K_{3}, P_{4}\right\}$-decomposition of $G^{\prime}$. The only $K_{3}$ 's in $G^{\prime}$ are those that belong to the co-fishes we inserted, so we only need to show that removing those co-fishes does not prevent us from adapting the decomposition of $G^{\prime}$ in order to obtain a $\left\{K_{1,3}, P_{4}\right\}$-decomposition $D$ of $G$. The proof is similar to that of the reverse direction of Lemma 3, with the following modification: since $G$ is $K_{3}$-free, we have $N_{G}(u) \cap N_{G}(w)=\emptyset$, so if $\{u, w\}$ is the extremal edge of a $P_{4}$ in $D^{\prime}$, then it will map onto $\{v, w\}$ in $G$, where it will become the extremal edge of a $P_{4}$ in $D$ (as opposed to, possibly, a $K_{3}$ in the proof of Lemma 3 ).

We give a reduction from the following NP-complete variant of SAT [1]]:

MONOTONE NOT-ALL-EQUAL 3-SATISFIABILITY

Input: $\quad$ a Boolean formula $\phi=C_{1} \wedge C_{2} \wedge \cdots \wedge C_{n}$ without negations over a set $\Sigma=\left\{x_{1}, x_{2}, \ldots, x_{m}\right\}$, with exactly three distinct variables per clause.

Question: does there exist an assignment of truth values $f: \Sigma \rightarrow\{$ TRUE, FALSE $\}$ such that exactly one or two literals are TRUE in every clause of $\phi$ ?

Theorem 4. $K_{3}$-Free $\left\{K_{1,3}, P_{4}\right\}$-DECOMPOSition With MARKed Edges $i s$ $\mathrm{NP}$-complete. 
Proof. Given an instance $\phi$ of MONOTONE NOT-ALL-EQUAL 3-SATISFIABILITY, we build an instance $(G, M)$ of $K_{3}$-FREE $\left\{K_{1,3}, P_{4}\right\}$-DECOMPOSITION With MARKED EDGES as follows:

1. For each variable $x_{i}$ with $k$ occurrences (we can assume $k \geq 2$ ), we create a tree $T\left(x_{i}\right)$ with $3 k$ leaves, called a variable tree, whose edges are all marked (see Figure 5(a)). Edges incident to a leaf are called border edges, the others are called internal edges.

2. For each clause $x_{i} \vee x_{j} \vee x_{k}$, we create a $P_{7}\left(v_{1}, v_{2}, \ldots, v_{7}\right)$, called a clause path, with marked edges $\left\{v_{2}, v_{3}\right\}$ and $\left\{v_{5}, v_{6}\right\}$, to which we join three border edges of each variable tree as follows (see Figure 6):

- one leaf of $T\left(x_{1}\right)$ is joined to $v_{1}$, another to $v_{3}$, and another to $v_{7}$;

- one leaf of $T\left(x_{2}\right)$ is joined to $v_{1}$, another to $v_{5}$, and another to $v_{7}$;

- one leaf of $T\left(x_{2}\right)$ is joined to $v_{2}$, another to $v_{4}$, and another to $v_{6}$.

Note that the resulting graph is indeed cubic: each inner vertex of a variable tree has degree 3, and each leaf is also part of a clause path. Furthermore, the inner vertices of a clause path are adjacent to two other vertices in the path and one other vertex in a variable tree, and each endpoint of a clause path is adjacent to another vertex in the path and two vertices in different variable trees.

We further observe that $G$ is $K_{3}$-free. First, any cycle included only in variable trees has length at least 4 (since it must be included in at least 2 such tree, and in each tree the path between any pair of leaves has length at least 2). Therefore, a $K_{3}$ would have to use 1 or 2 edges in a clause path. If it uses only one edge $\left\{v_{i}, v_{i+1}\right\}$, then both $v_{i}$ and $v_{i+1}$ must be joined to leaves of the same variable tree, which is impossible since each clause consists of three different variables. If two edges are used, then the last edge of the $K_{3}$ would be joining two leaves in some variable tree, which is also impossible. Therefore, $G$ is $K_{3}$-free.

We now prove that $\phi$ is satisfiable iff $(G, M)$ admits a decomposition.

$\Rightarrow$ : From a satisfying truth assignment, we create a $\left\{K_{1,3}, P_{4}\right\}$-decomposition of $(G, M)$ as described in Figures $5(b-c)$ and $6(b-e)$. Specifically, for each variable $x_{i}$ with $k$ occurrences, if $x_{i}=$ TRUE, then we cover all edges of $T\left(x_{i}\right)$ with $2 k-1 K_{1,3}$ 's (Figure $5(b)$ ). If $x_{i}=$ FALSE, then we cover all internal edges of $T\left(x_{i}\right)$ with $k-1 K_{1,3}$ 's(Figure $5(c)$ ).

Now for any clause $x_{i} \vee x_{j} \vee x_{k}$, at least 1 and at most 2 variables among $\left\{x_{i}, x_{j}, x_{k}\right\}$ are set to FALSE. For these two variables and the corresponding variable trees, the border edges are still uncovered (for variables assigned TRUE, border edges are covered with the rest of the variable tree). Each tree has 3 border edges coming to the clause path, so there are either 9 or 12 edges to cover ( 6 in the clause path and 3 or 6 in border variable trees). As shown in Figures $5(b-e)$, there always exist a decomposition of these edges into $K_{1,3}$ 's and $P_{4}$ 's (with the constraint that no marked edge is the middle of a $\left.P_{4}\right)$.

Overall, all edges in all variable trees and clause paths are covered by a $K_{1,3}$ or a $P_{4}$, therefore $(G, M)$ admits a $\left\{K_{1,3}, P_{4}\right\}$-decomposition. 


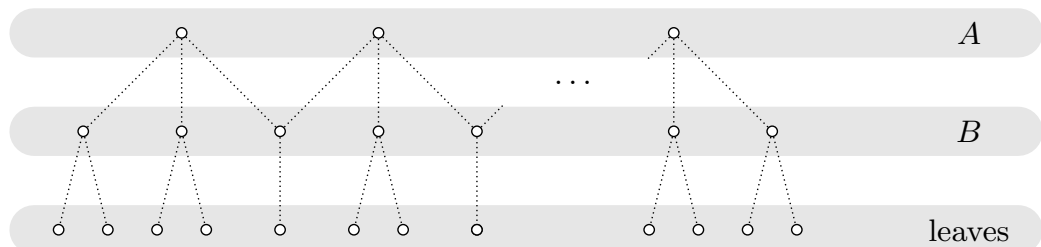

(a)

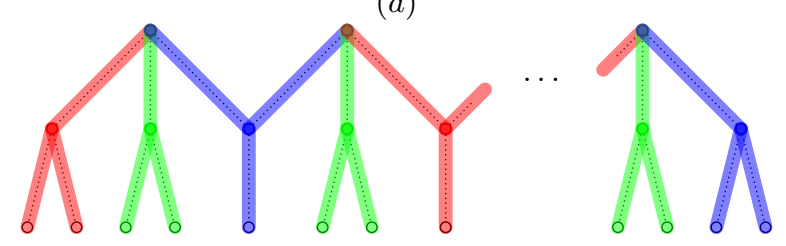

(b)

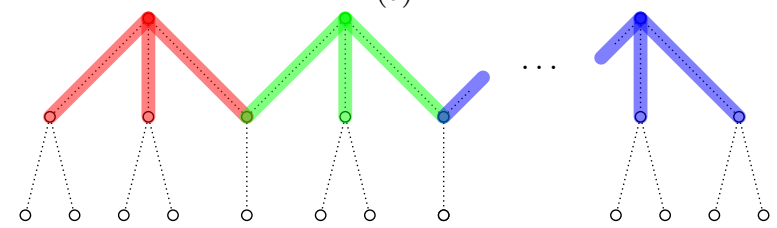

$(c)$

Fig. 5. (a) A variable tree $T\left(x_{i}\right)$ for a variable $x_{i}$ with $k$ occurrences: all its edges are marked, and it has $3 k$ leaves. Internal vertices are partitioned into two sets $A$ and $B$. (b) A decomposition of $T\left(x_{i}\right)$, corresponding to $x_{i}=$ TRUE. (c) A decomposition of the internal edges of $T\left(x_{i}\right)$, corresponding to $x_{i}=$ FALSE.

$\Leftarrow$ : We first consider any variable tree, and show that the decompositions used for TRUE and FALSE assignments above are in fact the only two possible decompositions of the internal edges of this tree. Indeed, consider internal vertices and their partition into $A$ and $B$ (see Figure $5(a)$ ): because of marked edges, all edges between any two internal vertices must be part of a $K_{1,3}$, linking a leaf to a center. Since internal vertices form a path, they must alternate along this path between leaves and centers of $K_{1,3}$ 's, so either all vertices of $B$ are centers, or all vertices of $A$ are centers. Each case yields only one possible decomposition of adjacent edges, as described respectively in Figures $5(b)$ and $5(c)$. Naturally, we assign true to any variable whose tree is decomposed as in the first case, and FALSE to other variables. We further make the following observation for the FALSE case: consider any leaf $x$ of the tree, and its parent $y$. Due to marked edges, $x$ can only be the center of a $K_{1,3}$, or a middle node of a $P_{4}$, of which $y$ is an endpoint.

We now consider a clause $x_{i} \vee x_{j} \vee x_{k}$, and show that its variables can neither be all set to TRUE nor all set to FALSE. Aiming at a contradiction, assume first that all variables are set to TRUE. Then all border edges of their trees are already covered, and the $K_{1,3}$ 's and $P_{4}$ 's covering the clause path may only use the 6 edges of the path. The only possibility to decompose the path in such a way is to use two $P_{4}$ 's, however, such $P_{4}$ 's would have marked 


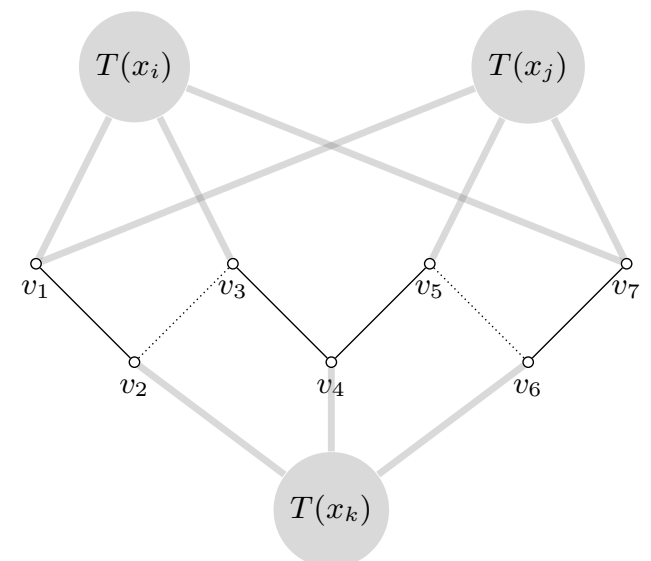

(a)
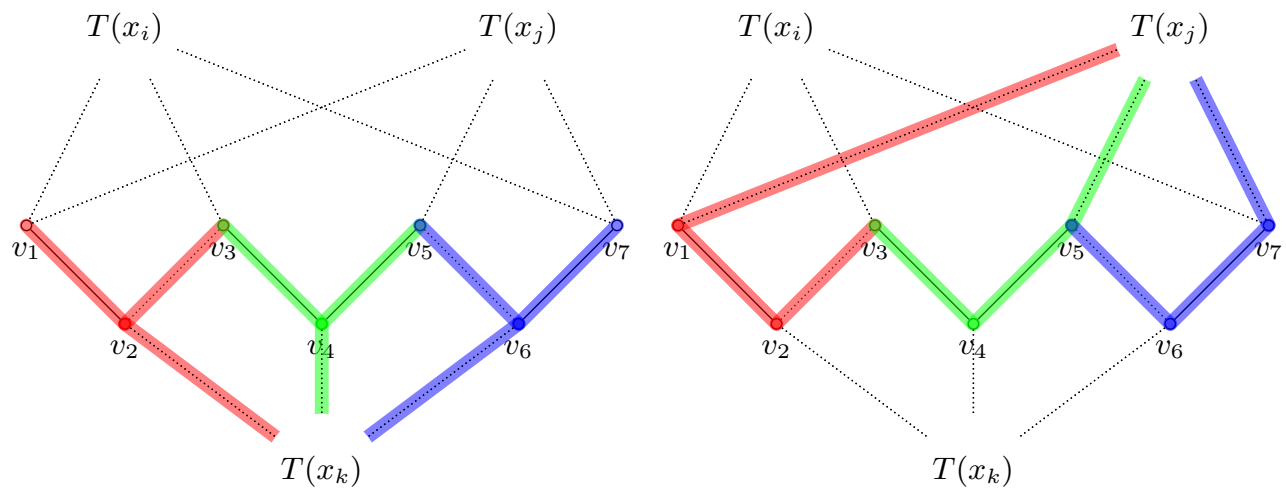

(b) $x_{i}=$ TRUE, $x_{j}=$ TRUE,$x_{k}=$ FALSE

(c) $x_{i}=$ TRUE, $x_{j}=$ FALSE, $x_{k}=$ TRUE

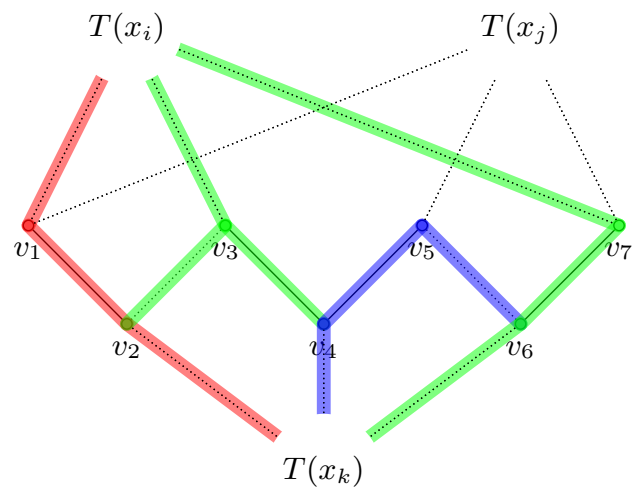

(d) $x_{i}=$ FALSE,$x_{j}=$ TRUE,$x_{k}=$ FALSE

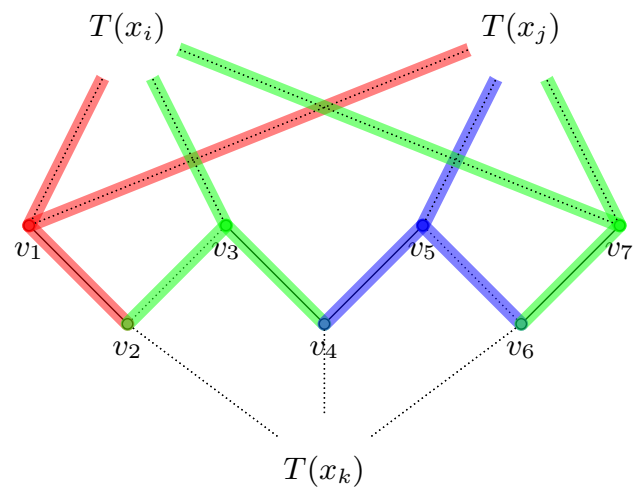

(e) $x_{i}=$ FALSE,$x_{j}=$ FALSE,$x_{k}=$ TRUE

Fig. 6. (a) A clause path and its connections to the variable trees. $(b-e)$ For each truth assignment of $x_{i}, x_{j}, x_{k}$ (up to symmetry), a decomposition of the path edges and the neighboring uncovered edges of variable trees for false variables. 
edges as middle edges, which is forbidden. We now assume that all variables of a clause are set to FALSE, i.e. it remains to cover the clause path and all border edges of the variable trees. Thanks to the observation made on leaves of the tree in the FALSE case, vertices $v_{1}$ and $v_{2}$ are either centers of $K_{1,3}$ 's, or middle nodes in $P_{4}$ 's. They cannot both be centers of $K_{1,3}$ 's, and due to marked edges, they must both be middle nodes of the same $P_{4}$. However, as noted above, the parents of $v_{1}$ in both trees $T\left(x_{i}\right)$ and $T\left(x_{j}\right)$ should be endpoints of this $P_{4}$, which is impossible.

Finally, each variable has been assigned a truth value, and for each clause there must be at least one TRUE and one FALSE variable: therefore, we have a satisfying assignment for our instance of MONOTONE NOT-ALL-EQUAL 3SATISFIABILITY. 